\title{
Two different modes associated with the composite nucleus - the charge and mass distributions for exotic nuclear synthesis -
}

\author{
Yoritaka Iwata $^{1, a}$, Takaharu Otsuka ${ }^{2,3}$, Joachim A. Maruhn $^{4}$, and Naoyuki Itagaki ${ }^{2}$ \\ 1 GSI Helmholtzzentrum für Schwerionenforschung, D-64291 Darmstadt, Germany \\ 2 Department of Physics, University of Tokyo, Hongo, Tokyo 113-0033, Japan \\ 3 Center for Nuclear Study, University of Tokyo, Hongo, Tokyo 113-0033, Japan \\ 4 Institut für Theoretische Physik, Universität Frankfurt, D-60325 Frankfurt, Germany
}

\begin{abstract}
The mass-dependent structure of the composite nucleus is shown based on three-dimensional timedependent Hartree-Fock calculations with Skyrme interactions (SLy4d and SkM*). One remarkable result is that the isovector monopole excitation dominantly appears for collisions of heavy nuclei, and the isovector dipole excitation for those of light ones. Such a difference found in the dynamical structure of composite nucleus plays a role in the equilibration of charge.
\end{abstract}

\section{Introduction}

If one wants to have a synthesis of a nucleus with a certain charge and mass, it is necessary to find factors to control charge and mass distributions of final fragments. The nuclear synthesis towards the neutron/proton driplines is one of the most interesting issues as for clarifying the existencelimit of elements. In this context, the ultimate goal is to obtain the control theory of nuclear synthesis from the view of collision dynamics.

The bombarding energy is a primary factor to control the $N / Z$ ratio of the final fragment $[1,2]$, hence the simple rule governing the appearance/disappearance of charge equilibration has been clarified to be essentially associated with the bombarding energy. Charge equilibration naturally prevents the synthesis of exotic nuclei with extreme proton-neutron asymmetry, therefore, its appearance is directly connected with the $N / Z$ ratios of the final fragments. Because the mass of final fragments becomes smaller by increasing the bombarding energy, there should be an upperlimit of the final mass yields for a given N/Z ratio. Another important factor arises from the choice of colliding nuclei, where the importance of such a choice to nuclear synthesis has been pointed out (for example, see [3]). Consequently, the bombarding energy, the total mass, and the protonneutron asymmetry are the three major factors (control parameters) of nuclear synthesis.

Most of all, the mass dependence of the collision dynamics has still been an outstanding issue to a large extent, where the fusion hindrance effect for collisions involving heavy nuclei was reported more than 20 years ago (for example, see [4-7]). In this paper we focus on the massdependent dynamics of collisions. The study is based on time-dependent Hartree-Fock (TDHF) calculations with Skyrm

\footnotetext{
a e-mail: y.iwata@gsi.de
}
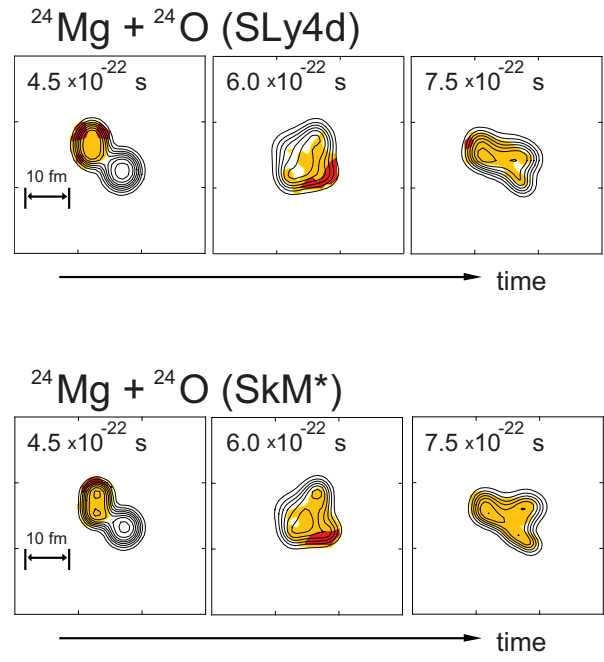

Fig. 1. Real-time dynamics on the reaction plane. Charge distributions of ${ }^{24} \mathrm{Mg}+{ }^{24} \mathrm{O}$ for the bombarding energy $18 \mathrm{MeV} / \mathrm{A}$ (laboratory frame) and the impact parameter $5.0 \mathrm{fm}$ are shown. ${ }^{24} \mathrm{Mg}$ is incoming from the left, and ${ }^{24} \mathrm{O}$ from the right. The colored regions show the distribution of charge (relatively proton-rich part), and the contours show the density, where parts with the proton to neutron density ratio larger than 1.00 and 0.80 are colored in red and yellow, respectively. For reference, a single time period of the isovector dipole oscillation of the total system $\left({ }^{48} \mathrm{Ca}\right)$ is estimated to be $1.97 \times 10^{-22} \mathrm{~s}$ using $\hbar \omega=77 A^{-1 / 3}$. The Skyrme TDHF with SLy4d parametrization is shown in the upper panel, and that with $\mathrm{SkM}^{*}$ parametrization in the lower panel.

interactions, where we show calculations with two different Skyrme parameter sets (SLy4d and SkM*) in order to see the robustness of the TDHF results.

This is an Open Access article distributed under the terms of the Creative Commons Attribution-Noncommercial License, which permits unrestricted use, distribution, and reproduction in any noncommercial medium, provided the original work is properly cited. 

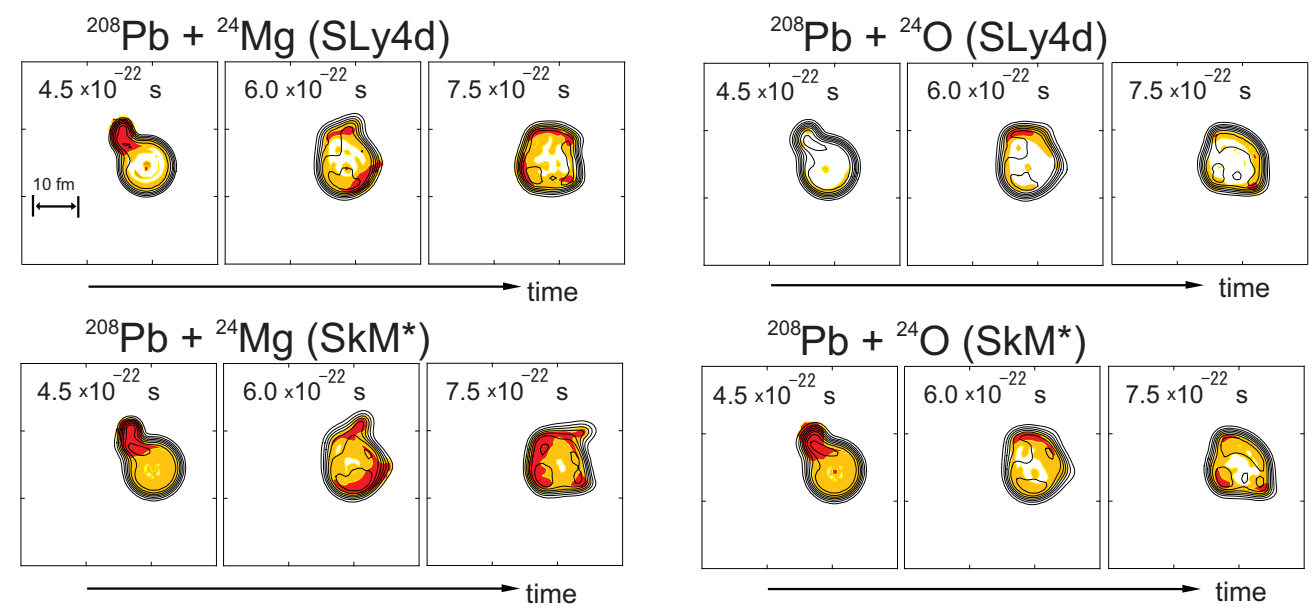

Fig. 2. (Color online) Real-time dynamics on the reaction plane. Charge distributions of ${ }^{208} \mathrm{~Pb}+{ }^{24} \mathrm{Mg}$ and ${ }^{208} \mathrm{~Pb}+{ }^{24} \mathrm{O}$ for the bombarding energy $21.6 \mathrm{MeV} / \mathrm{A}$ (laboratory frame) and the impact parameter $7.5 \mathrm{fm}$ are shown. ${ }^{24} \mathrm{Mg}$ and ${ }^{24} \mathrm{O}$ are incoming from the left, and ${ }^{208} \mathrm{~Pb}$ from the right. The colored regions show the distribution of charge (relatively proton-rich part), and the contours show the density, where parts with the proton to neutron density ratio larger than 0.75 and 0.70 are colored in red and yellow, respectively.

\section{Mass-dependent dynamics}

This section is organized as follows: the mass dependence at lower energies is developed in Sec. 2.1, that at higher energies in Sec. 2.2, and those features dependent only weakly on energy in Sec. 2.3.

\subsection{Two isovector modes}

In lower energy collisions (roughly speaking, less than 40 $\mathrm{MeV} / \mathrm{A}$ in the laboratory frame ${ }^{1}$ ), the charge equilibrium is predominantly achieved. In such cases, the equilibrium of charge during the collisions was shown to be mainly due to the collective motion highly associated with the meanfield [2]. Therefore, in this section, special attention is paid to the isovector collective motions.

Let us begin with a collision between light nuclei. Figure 1 shows the Skyrme TDHF dynamics of charge distribution for ${ }^{24} \mathrm{O}+{ }^{24} \mathrm{Mg}$, where two different parameter sets of Skyrme TDHF calculations are demonstrated. We see the appearance of the isovector dipole (iv-dipole) oscillation of well-localized charge $\left(4.5,6.0 \times 10^{-22} \mathrm{~s}\right)$ independent of the parameter set. Charge equilibration is synchronized with the iv-dipole excitation (for time evolutions of the dipole mode, see [8])); we will find that this is true only for collisions between light nuclei. Note that a dipole oscillation of the total system for a few cycles is sufficient to attain charge equilibrium, which implies how rapid the charge equilibration is.

Next we move on to collisions including a heavier nucleus: ${ }^{208} \mathrm{~Pb}+{ }^{24} \mathrm{Mg}$ and ${ }^{208} \mathrm{~Pb}+{ }^{24} \mathrm{O}$, where ${ }^{24} \mathrm{Mg}(\beta$ stable nucleus) and ${ }^{24} \mathrm{O}$ (neutron-rich nucleus) are an isobaric pair of $\beta$-stable and neutron-rich nuclei, respectively.

\footnotetext{
$140 \mathrm{MeV} / \mathrm{A}$ corresponds to the upper energy-limit of charge equilibration, which is estimated from the standard value of Fermi momentum: $k_{F}=1.36 \mathrm{fm}^{-1}$.
}

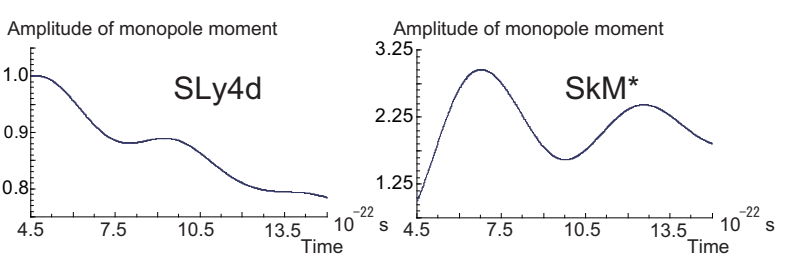

Fig. 3. Time evolution of monopole moment for ${ }^{208} \mathrm{~Pb}+{ }^{24} \mathrm{Mg}$. The monopole moment of a whole system is measured, and values are normalized by the value at $4.5 \times 10^{-22} \mathrm{~s}$ (almost equal to the contact time). For reference, the excitation energy is roughly estimated to be $7.0 \mathrm{MeV}$, because the time period of oscillation in this figure can be read as $6.0 \times 10^{-22} \mathrm{~s}$.

Figure 2 shows the TDHF calculations with a fixed impact parameter $(b=7.5 \mathrm{fm})$. The equilibration dynamics of charge is not similar to the motion of the iv-dipole excitation in these cases. Instead a spherical layered structure of the composite nucleus appears in both collisions $(7.5$ $\times 10^{-22} \mathrm{~s}$ ), in which a relatively neutron-rich core is in the center, a proton-rich layer surrounds it, and a neutronrich skin is in the surface. This is analogous to the isovector monopole (iv-monopole) excitation. Although TDHF results do not show any significant monopole oscillation in collisions involving light nuclei $\left({ }^{24} \mathrm{Mg}+{ }^{24} \mathrm{O}\right)$, there exist monopole excitations in the heavier cases (Fig. 3), where the damping in Fig. 3 is associated with the dynamics towards fusion. Fusion is achieved more rapidly in case of the SLy4 parametrization, while slowly in the $\mathrm{SKM}^{*}$ parametrization. The appearances of monopole oscillation seem to be due to the Coulomb repulsion, hence the spherical distribution of charge is formed. Note that the appearance of iv-monopole excitation is not dependent on the neutron-richness of the colliding nuclei.

The reaction dynamics (equivalent to the dynamics towards charge equilibrium in such lower energy collisions) is quite different depending on the total mass of the two 
Table 1. N/Z ratios of parts (a), (b) and (c) in Fig. 4 are shown, where the averaged N/Z values are shown for the part (a).

\begin{tabular}{lrrr}
\hline & (a) & (b) & (c) \\
\hline SLy4d & 1.64 & 1.42 & 1.47 \\
SkM $^{*}$ & 1.63 & 1.50 & 1.50 \\
\hline
\end{tabular}

colliding nuclei. The iv-dipole excitation is dominant for lighter cases, and the iv-monopole excitation alternatively appears for heavier cases. Although the relation between the charge equilibration and iv-dipole excitation has been pointed out previously (for example, see [9-14]), that between charge equilibration and the iv-monopole excitation is pointed out here for the first time.

The discrepancy between fusion and charge equilibration was reported to be due to a mass dependent collision dynamics, which was explained by the nuclear binding energy including the Coulomb energy [2]. It is associated with the two different modes.

\subsection{Pattern of fragmentation}

Let us move on to collisions with higher energies (roughly speaking, more than $40 \mathrm{MeV} / \mathrm{A}$ in the laboratory frame). The mass dependence of the reaction dynamics can be found not only in the structure of the composite nucleus but also in the geometry of fragmentation. Under the disappearance of charge equilibration, the final fragments with a specific pattern are obtained only for collisions involving heavy nuclei. This kind of mass-dependence only appears in higher energy collisions [2].

For collisions between heavy nuclei, localization of charge around the contact time (Fig. 4), which is due to the Coulomb excitation, strongly appears. For simplicity, let us restrict ourselves to fragmentations into three parts, where we have a separation into parts (a), (b) and (c) shown in Fig. 4. Table 1 shows the N/Z ratios of parts (a), (b) and (c). For both parameter sets of SLy4 and SkM*, we have more neutronrich fragments with almost zero velocity, and more protonrich fragments with finite-valued velocity in the center-ofmass frame. Such a pattern formation in higher energies is specific to collisions involving heavy nuclei. This study may contribute to the arrangement of detector system in experiments.

\subsection{Formation of jet}

Let us study the mass-dependent dynamics in its features that depend only weakly on energy. For collisions involving heavy nuclei, three-dimensional time-dependent theoretical calculations predict the possible formation of nonrelativistic jets in low-energy heavy-ion collisions, where the relative velocity of collision is estimated to be a quarter of light velocity [2].

Let us move on to collisions: ${ }^{208} \mathrm{~Pb}+{ }^{132} \mathrm{Xe}$ and ${ }^{208} \mathrm{~Pb}+$ ${ }^{132} \mathrm{Sn}$, where ${ }^{132} \mathrm{Xe}\left(\beta\right.$-stable nucleus) and ${ }^{132} \mathrm{Sn}$ (neutronrich nucleus) are an isobaric pair of $\beta$-stable and neutronrich nuclei, respectively. The calculated time evolutions

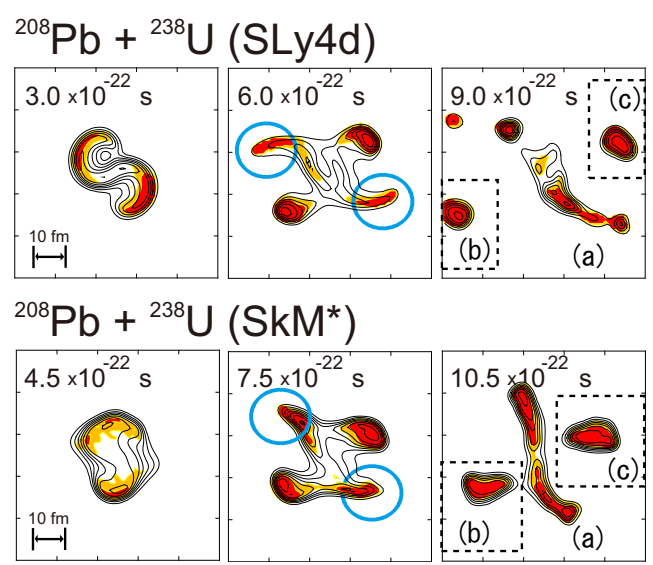

Fig. 4. (Color online) Real-time dynamics on the reaction plane. Charge distributions of ${ }^{208} \mathrm{~Pb}+{ }^{238} \mathrm{U}$ for the bombarding energy $32.1 \mathrm{MeV} / \mathrm{A}$ (laboratory frame) and the impact parameter $9.2 \mathrm{fm}$ are shown, where the upper energy-limit of charge equilibration is estimated to be $27.7 \mathrm{MeV} / \mathrm{A}$ (laboratory frame) based on the upper-limit formula $[1,2] .{ }^{238} \mathrm{U}$ is incoming from the left, and ${ }^{208} \mathrm{~Pb}$ from the right, where the blue circle designates the formation of jets. The description manner is the same as Fig. 2.

of the two reactions ${ }^{208} \mathrm{~Pb}+{ }^{132} \mathrm{Xe}$ and ${ }^{208} \mathrm{~Pb}+{ }^{132} \mathrm{Sn}$ are shown in Fig. 5, which also shows the formation of the layered structure with a neutron-rich core. Because the Coulomb energy becomes much higher compared to the reactions not involving heavier nuclei, the neutron-rich core formation appears more strongly. Coulomb excitation causes the localization of charge around the contact time $\left(4.5 \times 10^{-22} \mathrm{~s}\right)$, the neutron-rich core is formed $\left(6.0 \times 10^{-22} \mathrm{~s}\right)$, and the jet with velocity opposite or perpendicular to the relative velocity is formed (blue circles in $7.5 \times 10^{-22} \mathrm{~s}$ ). Formation of jets at a bombarding energy higher than the upper energy-limit of charge equilibration is shown as blue circles in Fig. 4, and that at a bombarding energy lower than the upper limit as blue circles in Fig. 5.

The formation of jets appears in calculations of collisions involving heavy nuclei (jets appear in ${ }^{208} \mathrm{~Pb}+{ }^{238} \mathrm{U}$, ${ }^{132} \mathrm{Xe},{ }^{132} \mathrm{Sn}$ in our calculations), and jets are clearly protonrich. It means that the origin of jets in low-energy heavyion collisions is the Coulomb repulsion, because there is no other reason to have such an aggregation dynamics of charge. Note that the disappearance of charge equilibration is not a necessary condition for the jet formation, therefore jet formation and the resulting nuclear emission are efficient ways to reach the equilibrium of charge. The jet is also meaningful enough to be an origin of rare nuclei.

\section{Conclusion}

In this paper, the mass-dependence of reaction dynamics is shown based on the three-dimensional time-dependent Hartree-Fock calculations with two Skyrme interactions (SLy4d and SkM*). For the physics discussed here, the TDHF results are independent of the two parameter sets. 

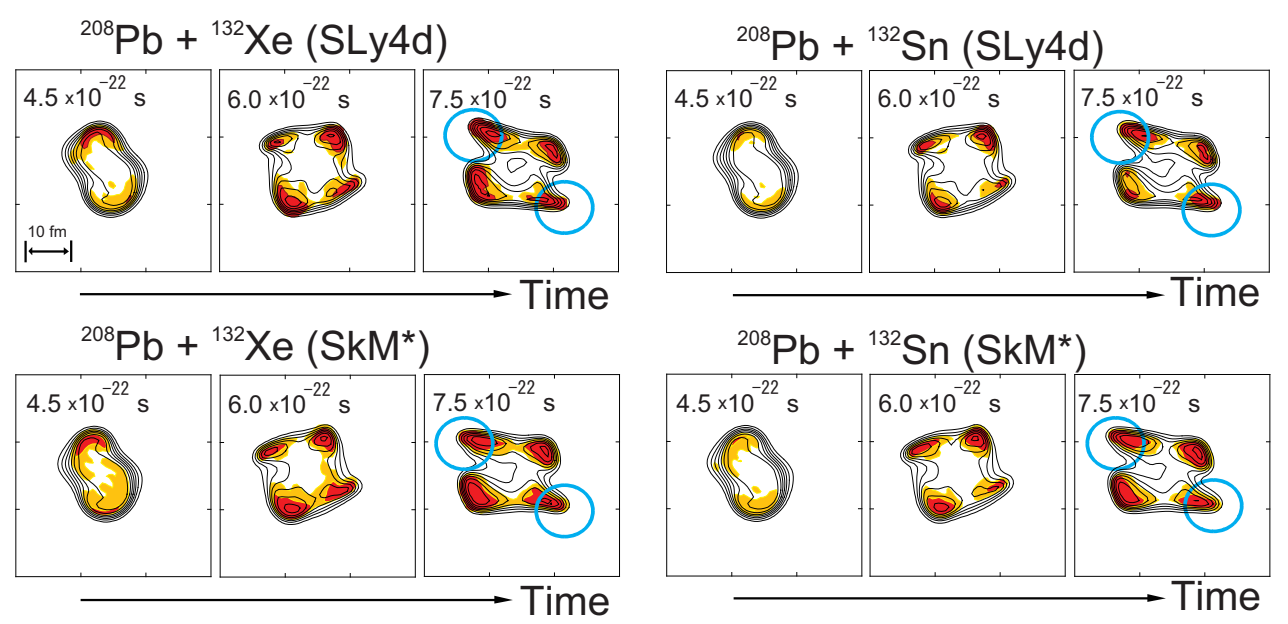

Fig. 5. (Color Online) Real-time dynamics on the reaction plane. Charge distributions of ${ }^{208} \mathrm{~Pb}+{ }^{132} \mathrm{Xe}$ and ${ }^{208} \mathrm{~Pb}+{ }^{132} \mathrm{Sn}$ for the bombarding 25.3 MeV/A (laboratory frame) and impact parameter $7.5 \mathrm{fm}$ are shown. ${ }^{132} \mathrm{Xe}$ and ${ }^{132} \mathrm{Sn}$ are incoming from the left, and ${ }^{208} \mathrm{~Pb}$ from the right. The blue circles designate the formation of jets. The description manner is the same as Figs. 2 and 4.

First of all, the mass-dependent structure of composite nucleus is shown. It is prominent here that the isovector monopole excitation appears for collisions involving a heavy nucleus, and the isovector dipole excitation for those including light ones. Such a difference is directly connected to the different type of charge equilibration dynamics. Next, massdependent pattern formation of final fragments is shown. In particular, a specific pattern of fragmentation, in which the neutron-rich fragments tend to be produced with zero velocity in the center-of-mass frame, is achieved only in reactions involving heavy nuclei. Finally, the formation of non-relativistic jets in low-energy heavy-ion collisions is predicted based on three-dimensional TDHF results. As is quite natural, all three kinds of mass-dependence can be regarded as different appearances of the Coulomb excitation for light and heavy nuclear systems.

This work was supported by EMMI of the Helmholtz Alliance, JSPS Core-to-Core Program EFES, and the TodaiRIKEN Joint International Program for Nuclear Physics (TORIJIN).

\section{References}

1. Y. Iwata, T. Otsuka, J. A. Maruhn, and N. Itagaki, AIP Conference Proceedings 1098 (2009) 308.

2. Y. Iwata, T. Otsuka, J. A. Maruhn, and N. Itagaki, to appear in Euro. Phys. J. A.

3. V. Zagrebaev and W. Greiner, Phys. Rev. Lett. 101 (2008) 122701.

4. W. J. Swiatecki, Nucl. Phys. A376 (1982) 275.

5. S. Bjornholm and W. J. Swiatecki, Nucl. Phys. A391 (1982) 471.

6. Y. Abe et. al., Physics of Atomic Nuclei, 66 (2003) 1057.
7. Y. Abe and B. Bouriquet, Acta Phys. Pol. B34 (2003) 1927.

8. Y. Iwata, T. Otsuka, J. A. Maruhn, and N. Itagaki, in preparation.

9. M. Berlanger, A. Gobbi, F. Haneppe, U Lynen, C. Ngô and A. Olmi, Z. Phys. A 291 (1979) 133.

10. E. S. Hernandez, W. D. Myers, J. Randrup and B. Remaud, Nucl. Phys. A 361 (1981) 483.

11. P. Bonche and N. Ngô, Phys. Lett. B 105 (1981) 17.

12. E. Suraud, M. Pi and, P. Schuck Nucl. Phys. A 492 (1989) 294.

13. C. Simenel, Ph. Chomaz, and G. de France, Pys. Rev. Lett. (2001) 2971.

14. C. Simenel, Ph. Chomaz, and G. de France, Pys. Rev. C 76 (2007) 024609. 